\title{
Spatial and temporal skin blood volume and saturation estimation using a multispectral snapshot imaging camera
}

Maria Ewerlöf, Marcus Larsson, E. Göran Salerud

Maria Ewerlöf, Marcus Larsson, E. Göran Salerud, "Spatial and temporal skin blood volume and saturation estimation using a multispectral snapshot imaging camera," Proc. SPIE 10068, Imaging, Manipulation, and Analysis of Biomolecules, Cells, and Tissues XV, 1006814 (16 February 2017); doi: $10.1117 / 12.2251928$

SPIE. Event: SPIE BiOS, 2017, San Francisco, California, United States 


\title{
Spatial and temporal skin blood volume and saturation estimation using a multispectral snapshot imaging camera
}

\author{
Maria Ewerlöf, Marcus Larsson, E. Göran Salerud \\ Department of Biomedical Engineering, Linköping University, S-581 83 Linköping, Sweden
}

\begin{abstract}
Hyperspectral imaging (HSI) can estimate the spatial distribution of skin blood oxygenation, using visible to near-infrared light. HSI oximeters often use a liquid-crystal tunable filter, an acousto-optic tunable filter or mechanically adjustable filter wheels, which has too long response/switching times to monitor tissue hemodynamics. This work aims to evaluate a multispectral snapshot imaging system to estimate skin blood volume and oxygen saturation with high temporal and spatial resolution.

We use a snapshot imager, the xiSpec camera (MQ022HG-IM-SM4X4-VIS, XIMEA®), having 16 wavelength-specific Fabry-Perot filters overlaid on the custom CMOS-chip. The spectral distribution of the bands is however substantially overlapping, which needs to be taken into account for an accurate analysis.

An inverse Monte Carlo analysis is performed using a two-layered skin tissue model, defined by epidermal thickness, haemoglobin concentration and oxygen saturation, melanin concentration and spectrally dependent reduced-scattering coefficient, all parameters relevant for human skin. The analysis takes into account the spectral detector response of the xiSpec camera. At each spatial location in the field-of-view, we compare the simulated output to the detected diffusively backscattered spectra to find the best fit. The imager is evaluated for spatial and temporal variations during arterial and venous occlusion protocols applied to the forearm. Estimated blood volume changes and oxygenation maps at $512 \times 272$ pixels show values that are comparable to reference measurements performed in contact with the skin tissue. We conclude that the snapshot xiSpec camera, paired with an inverse Monte Carlo algorithm, permits us to use this sensor for spatial and temporal measurement of varying physiological parameters, such as skin tissue blood volume and oxygenation.
\end{abstract}

Keywords: Multispectral imaging; hyperspectral imaging; diffuse reflectance spectroscopy; Monte Carlo simulations; computer modelling and simulation; skin blood saturation; microcirculation

\section{INTRODUCTION}

The oxygenation of living tissue is a fundamental parameter to measure for evaluating the homeostasis of cells and tissue ${ }^{1-3}$. Clinically, visual observations of temporal and spatial skin colour variations, as a reaction to provocation, can be used to indirectly assess the microcirculatory blood perfusion and oxygenation ${ }^{4,5}$. Visual assessment is based on the unique and well-defined light absorbing properties of haemoglobin, a dominant chromophore found in red blood cells, where the haemoglobin spectral signature depends on the degree of oxygen saturation ${ }^{6}$. In the diffuse reflectance spectrum from human skin, concentrations of dominant chromophores in skin tissue (i.e., oxygenated haemoglobin, deoxygenated haemoglobin, and melanin) are mirrored, and possible to resolve ${ }^{7-9}$.

Standard diffuse reflectance spectroscopy (DRS) utilize optical fiber probes with a well-defined distance between sending and receiving fibers ${ }^{10}$. In the visible spectrum (VIS) range, gratings and CCD arrays are used for recording DRS-data having more than 2000 equidistant wavelength bands in the range 450 to $700 \mathrm{~nm}$. Calculation of blood volume and oxygenation may be performed using diffusion theory ${ }^{11}$, inverse Monte Carlo modelling ${ }^{12-14}$ or hybrid models ${ }^{15}$. To extend DRS into spatial mapping of the chromophores in skin tissue, multi- or hyper-spectral imaging (HSI) techniques may be needed, and are possible to implement by a monochromatic CCD camera with narrow band filters and a white light source ${ }^{16-20}$ or using modulated light sources ${ }^{21}$.

Imaging, Manipulation, and Analysis of Biomolecules, Cells, and Tissues XV, edited by

Daniel L. Farkas, Dan V. Nicolau, Robert C. Leif, Proc. of SPIE Vol. 10068, 1006814

(c) 2017 SPIE · CCC code: 1605-7422/17/\$18 - doi: 10.1117/12.2251928 
Due to the high dimensional nature of spectral data, many classical algorithms in pattern recognition and machine learning have naturally been borrowed and adapted to perform feature extraction and classification and to shorten the calculation time ${ }^{22-24}$. To accurately analyse large high-resolution HSI datasets, using complex light transport models to quantify the blood volume and oxygenation, is however computationally demanding. Using such methods for on-line mapping of changes in physiology are extremely challenging. To lower the computational complexity, reduced HSI datasets may be used. This can be achieved using only a selected number of wavelengths (multispectral imaging, MSI) or by using multiple cameras with properly selected filters ${ }^{19,25}$. By wisely selecting a subset of wavelengths, the estimation accuracy can be pertained ${ }^{26-28}$. Even though the number of wavelengths can be reduced, many MSI techniques still suffer from a slow acquisition speed. This includes set-ups using liquid-crystal tunable filters, acousto-optic tunable filters or mechanically adjustable filter wheels. The slow acquisition speed can be overcome using custom matrix filters placed directly on-top of an imaging sensor for instantaneously acquisition of spatial and spectral data ${ }^{29}$. The development of such image sensor technology has made it possible to capture MSI data in a few spectral bands where the haemoglobin molecule exhibits strong characteristic spectral features. This is implemented in the xiSpec MSI snapshot imager (XIMEA ${ }^{\circledR}$, Germany), having 16 wavelength-specific Fabry-Perot filters overlaid on a custom CMOS-chip enabling up to 170 data cubes to be captured every second. However, the spectral sensitivity distribution of the bands is substantially overlapping and partially multimodal, which needs to be taken into account for an accurate analysis of tissue blood volume and oxygenation. Therefore, to create spatial blood volume and oxygenation maps of tissue, we need to enhance and develop the analysis and modelling of reduced MSI datasets.

The aim of this study is to evaluate if spatial and temporal variations in blood volume and oxygenation can be accurately estimated from data captured using the xiSpec camera, using an inverse Monte Carlo algorithm. Temporal data is acquired during an occlusion-release test on the lower forearm of one test subject.

\section{METHODS}

\subsection{Measurement set-up}

The measurement set-up includes a xiSpec camera (MQ022HG-IM-SM4X4-VIS, XIMEA®, Germany). It has 16 wavelength-specific Fabry-Perot filters in the 470-630 nm range (see Figure 1, upper panel) overlaid on a custom CMOSchip (2048x1088 pixels) in a mosaic pattern. To reduce noise, the xiSpec camera takes 16 consecutive snapshots (12 bit) that are summed up to one image before being stored. This gives the MSI system an effective framerate of 1.4 fps and an image depth of 16 bit. The light source used is an eight LED ring light (R130, Smart Vision Lights, USA) emitting white light with a known spectrum, (Figure 1, lower panel). The xiSpec camera is mounted side by side to the ring light and both light source and camera are equipped with polarization filters in front, to minimize specular reflections.

A modified PF6000 instrument (Perimed AB, Sweden), containing a probe based, spatially resolved, DRS system, is used for reference measurements of pointwise skin blood volume and oxygenation. The instrument, denoted EPOS (Enhanced Perfusion and Oxygen Saturation monitor), contains two spectroscopic channels collecting backscattered light in the 450$850 \mathrm{~nm}$ range at two different source-detector separations. The acquired spectra are analysed using a refined inverse Monte Carlo algorithm based on a three-layered bio-optical skin tissue model ${ }^{30}$.

\subsection{Provocation protocol}

The provocation protocol includes measurements for a period of twenty minutes; five minutes of baseline before occlusion onset, five minutes of occlusion and thereafter ten minutes of recovery. Occlusion of the forearm is performed by applying a cuff around the upper arm of the test subject. The cuff is automatically, and quickly, inflated to a pressure of $250 \mathrm{mmHg}$ for arterial occlusion and $45 \mathrm{mmHg}$ for venous occlusion. The cuff maintain a constant pressure for five minutes, and is then automatically deflated to release the occlusion. The arterial occlusion protocol is performed first and one hour after the protocol is finished, the venous occlusion protocol is performed on the same arm. The measurement protocols on human test subjects have been reviewed and approved by the Regional Ethical Review Board in Linköping (Dnr 2015/392-31).

Before the measurement starts, the test subject has been acclimatized to room temperature for at least 15 minutes. The test subject is asked to sit comfortably in a chair with the lower arm placed on a stable surface $30 \mathrm{~cm}$ below the xiSpec camera. A vacuum pillow is used as support and fixation, which makes it easier for the test subject to relax and keep the arm steady throughout the measurements. A square pattern $(4 \times 4 \mathrm{~cm})$, outlined with nine dots, is used as spatial position reference, and marked with ink on arm. The probe of the EPOS system is placed close to, but outside, the marked area. 

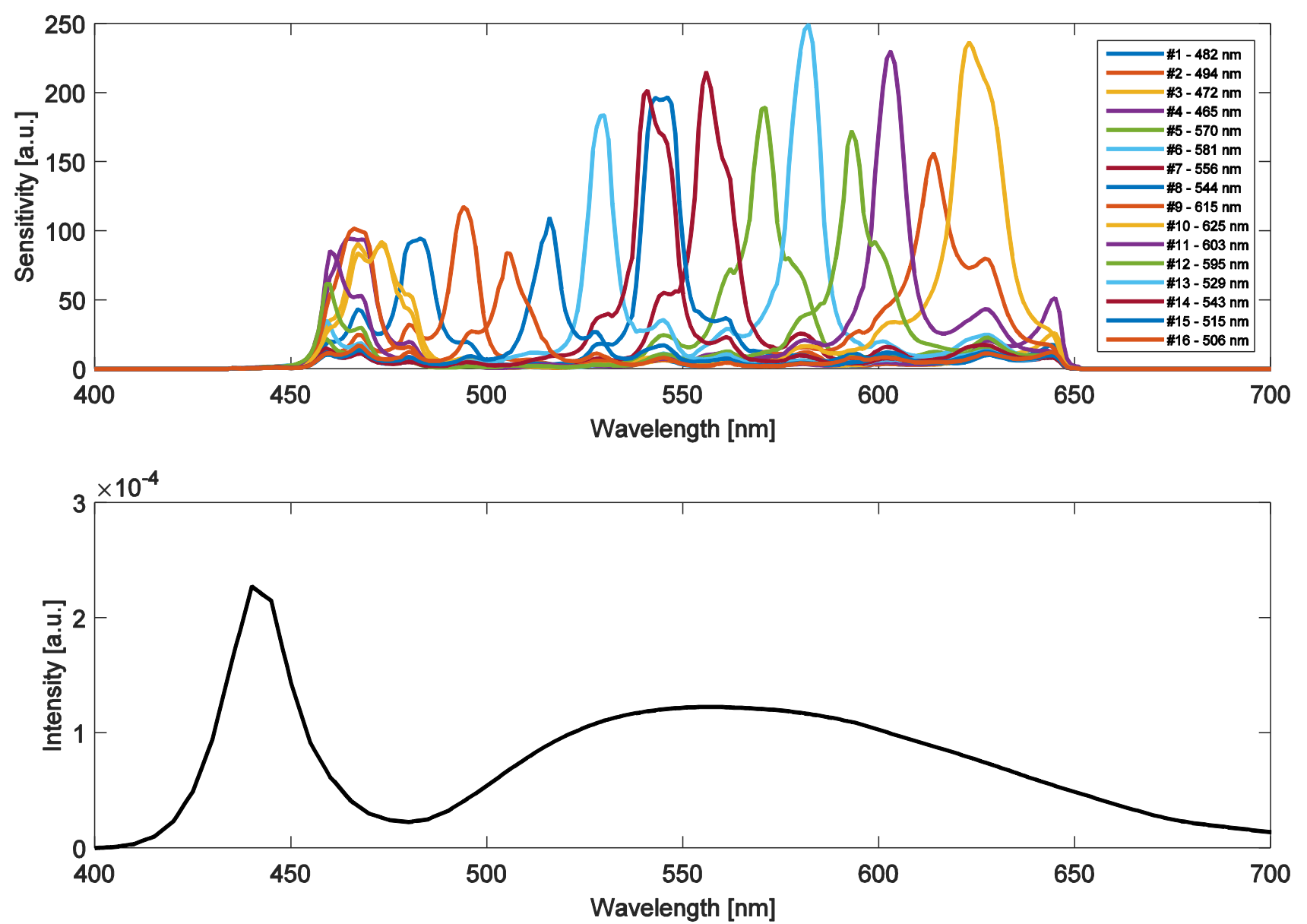

Figure 1. Upper panel: The manufacturer's data of detector sensitivity for the 16 channels of the xiSpec camera. Lower panel: The manufacturer's data of emitted spectra from the LED ring light.

The light source is turned on during measurements except for three ten second periods when dark calibration data is acquired; 4 minutes into the baseline phase ( $\mathrm{t}=4$ minutes), 4 minutes into the occlusion phase $(\mathrm{t}=9$ minutes $)$ and 9 minutes into the re-perfusion phase $(\mathrm{t}=19$ minutes). In parallel to the image acquisition the EPOS system measures continuously throughout the complete experiment.

After both provocation protocols are finished, a white reference tile Spectralon ${ }^{\circledR}$ (LabSphere, USA) is placed at the same location as the arm. Data is acquired for ten seconds with the ring light turned on and ten seconds with the ring light turned off. This data is used for dark image adjustment and white calibration during post processing of the measurements.

\subsection{Image processing}

The spatial data acquired by the xiSpec camera are spectrally sorted, generating an MSI image (hypercube) with an image format of (512x272 pixels), and a spectral depth of 16 wavelength bands. The MSI data, $I_{\mathrm{x}}(n)$, has 16 wavelength-specific channels, where $\mathrm{n}$ is the channel number. Each channel has its own spectral distribution with at least one main peak (Figure 1 , upper panel) and some overlap or have a multimodal distribution.

For temporal measurements, an area of interest $(2 \times 2 \mathrm{~cm})$ is tracked and extracted from all captured images. An average intensity spectrum is calculated over the area and used in the inverse Monte Carlo algorithm for estimation of blood volume and oxygenation. The spatial analysis is done pixel-by-pixel on the image of the lower arm after masking off the background. 
Dark calibration measurements are performed three times during the protocol. For each channel of the xiSpec camera, the average value of these three measurements is calculated and the resulting dark data cube, $I_{\mathrm{d}}(n)$, is subtracted from each acquired data cube.

A white normalization measurement is performed after each protocol is finished. The white normalization data is dark corrected as described above by $I_{\mathrm{d}, \mathrm{w}}(n)$, measured on the Spectralon ${ }^{\circledR}$ tile with the light source turned off. Multiple measurements are combined where the tile position is changed since the Spectralon ${ }^{\circledR}$ is too small to cover the whole field of view. The white normalized data, $I_{\mathrm{x}}^{\mathrm{N}}(n)$, is given by

$$
I_{\mathrm{x}}^{\mathrm{N}}(n)=\frac{I_{\mathrm{X}}(n)-I_{\mathrm{d}}(n)}{I_{\mathrm{W}}(n)-I_{\mathrm{d}, \mathrm{w}}(n)} .
$$

\subsection{Blood volume and oxygenation estimation}

The inverse Monte Carlo algorithm, used for estimation of oxygen saturation and blood volume, is previously described in Ewerlöf et al, $2015^{26}$. It finds the best fit of a measured spectrum to a modelled spectra with known optical properties and assumes the same properties are relevant for the measurement. The modelled spectra are based on Monte Carlo simulations performed on a two-layered skin tissue model defined by six parameters, describing tissue scattering (parameter $\alpha$ and $\beta$ ), tissue fraction melanin $\left(c_{\text {mel }}\right)$, tissue fraction blood $\left(c_{\mathrm{b}}\right)$, blood oxygen saturation $\left(s_{\mathrm{b}}\right)$ and epidermal thickness $\left(T_{\text {epi }}\right)$. The parameters are used to calculate tissue optical properties; reduced scattering coefficient $\mu_{\mathrm{s}}^{\prime}(\lambda)$, melanin absorption coefficient $\mu_{\mathrm{a}, \mathrm{mel}}(\lambda)$ and blood absorption coefficient $\mu_{\mathrm{a}, \mathrm{b}}(\lambda)$ in accordance to Eqs. 2-4.

The scattering coefficient is described by a Henyey-Greenstein scattering phase function, with anisotropy factor $g=0.8$ for all wavelengths $\lambda$, via parameters $\alpha$ and $\beta$ according to Eq. ${ }^{31}$. Scattering properties are the same in both layers of the skin tissue model.

$$
\mu_{\mathrm{s}}^{\prime}(\lambda)=\alpha \cdot\left(\frac{\lambda}{500}\right)^{-\beta}
$$

The upper epidermal layer of the skin tissue model contains melanin, but no blood. The melanin absorption coefficient is given by Eq. $3^{31}$, where $c_{\text {mel }}$ is the tissue fraction of melanin. The thickness of the epidermal layer is described by parameter $T_{\text {epi }}$.

$$
\mu_{\mathrm{a}, \mathrm{mel}}(\lambda)=c_{\mathrm{mel}} \cdot 51.9 \cdot\left(\frac{\lambda}{500}\right)^{-3}
$$

The second layer is considered infinitely thick with regards to the optical properties. It contains blood, but no melanin and the absorption coefficient is given by Eq. 4 , where $s_{\mathrm{b}}$ is the oxygen saturation level, $c_{\mathrm{b}}$ is blood tissue fraction (blood volume), $\mu_{\mathrm{a}, \mathrm{Hb}}$ the absorption coefficient of deoxygenated haemoglobin, and $\mu_{\mathrm{a}, \mathrm{HbO} 2}$ the absorption coefficient of oxygenated haemoglobin ${ }^{6}$.

$$
\mu_{\mathrm{a}, \mathrm{b}}(\lambda)=\left(1-s_{\mathrm{b}}\right) \cdot c_{\mathrm{b}} \cdot 0.15 \cdot \mu_{\mathrm{a}, \mathrm{Hb}}(\lambda)+s_{\mathrm{b}} \cdot c_{\mathrm{b}} \cdot 0.15 \cdot \mu_{\mathrm{a}, \mathrm{HbO} 2}(\lambda)
$$

White Monte Carlo simulations are performed for discretized levels of $\mu_{\mathrm{s}}^{\prime}(\lambda)$ and $T_{\text {epi }}$. Absorption is added to each base simulation, using Beer-Lambert's law and respective path length in the epidermal layer and the second layer for each detected photon. The resulting intensities were saved in the look-up table $I_{\mathrm{LUT}}\left(\mu_{\mathrm{s}}^{\prime}, \mu_{\mathrm{a}, \mathrm{mel}}, \mu_{\mathrm{a}, \mathrm{b}}, T_{\mathrm{epi}}\right)$ and used to generate simulated diffuse reflectance spectra, $F^{\mathrm{MC}}(\lambda)$, using linear interpolation within the dataset. Figure 2 shows generated spectra for desaturated $\left(s_{\mathrm{b}}=0\right)$ and fully saturated $\left(s_{\mathrm{b}}=1\right)$ blood when all other parameters are set to their respective mid-value. The value range of each parameter is presented in Table 1. 
Table 1. The six parameters in the skin tissue model and their ranges. For the inverse Monte Carlo models (A and B), the values of the bound parameters are given by their interval mid-value.

\begin{tabular}{|c|c|c|c|}
\hline Parameter & Value range & Model A & Model B \\
\hline$\alpha$ & {$[3,6]$} & 4.5 & free \\
\hline$\beta$ & {$[0.5,2.5]$} & 1.5 & free \\
\hline$c_{\mathrm{mel}}$ & {$[0,0.084]$} & free & free \\
\hline$c_{\mathrm{b}}$ & {$[0,0.056]$} & free & free \\
\hline$s_{\mathrm{b}}$ & {$[0,1]$} & free & free \\
\hline$T_{\mathrm{epi}}$ & {$[50,200]$} & 125 & free \\
\hline
\end{tabular}

\subsubsection{Post processing of Monte Carlo simulated spectra}

The spectral distributions of the 16 channels are substantially overlapping (Figure 1, upper panel), which needs to be accounted for before comparing Monte Carlo modelled spectra to measured spectra in the inverse Monte Carlo algorithm. To compare a generated spectrum to the 16 channels measured by the xiSpec camera, the corresponding intensity, $I_{\mathrm{x}}^{\mathrm{MC}}(n)$, for each channel is calculated by Eq. 5. The xiSpec detector sensitivity $r_{\mathrm{n}}(\lambda)$ for each channel, $n$, is multiplied by the output spectrum, $L(\lambda)$, of the light source and the Monte Carlo modelled spectrum, $F_{\mathrm{x}}^{\mathrm{MC}}(\lambda)$. The total response for all wavelengths $(450-650 \mathrm{~nm})$ is summarized to get the total intensity response from each channel. The spectra of $r_{\mathrm{n}}(\lambda)$ and $L(\lambda)$ are presented in Figure 1.

$$
I_{\mathrm{x}}^{\mathrm{MC}}(n)=\sum_{\lambda} r_{\mathrm{n}}(\lambda) \cdot L(\lambda) \cdot F_{\mathrm{x}}^{\mathrm{MC}}(\lambda)
$$

The corresponding white normalization, $I_{\mathrm{w}}^{\mathrm{MC}}(n)$, is calculated by using the generated spectrum $F_{\mathrm{x}}^{\mathrm{MC}}(\lambda)=1$, as in Eq. 6 . Figure 3 shows $I_{\mathrm{w}}^{\mathrm{N}, \mathrm{MC}}(n)$ together with the corresponding intensities, $I_{\mathrm{w}}^{\mathrm{N}, \mathrm{M}}(n)$, measured with the xiSpec camera on the white Spectralon ${ }^{\circledR}$ tile.

$$
I_{\mathrm{w}}^{\mathrm{MC}}(n)=\sum_{\lambda} r_{\mathrm{n}}(\lambda) \cdot L(\lambda) \cdot 1
$$

White normalization intensity response, $I_{\mathrm{x}}^{\mathrm{N}, \mathrm{MC}}(n)$, from a Monte Carlo modelled spectrum is then given by Eq. 7 .

$$
I_{\mathrm{x}}^{\mathrm{N}, \mathrm{MC}}(n)=\frac{I_{\mathrm{X}}^{\mathrm{MC}}(n)}{I_{\mathrm{W}}^{\mathrm{MC}}(n)}
$$

\subsubsection{Inverse Monte Carlo algorithm}

To estimate skin blood oxygen saturation and blood volume from our measurements, inverse Monte Carlo calculations are used. Each measured spectrum is compared to simulated data with known optical properties. A trust-region-reflective algorithm (MATLAB version 8.2.0, 2013b, computer software, The MathWorks Inc., Natick, Massachusetts) is used to find the optimal fit between measured, $I_{\mathrm{x}}^{\mathrm{N}, \mathrm{M}}(n)$, and Monte Carlo modelled, $I_{\mathrm{x}}^{\mathrm{N}, \mathrm{MC}}(n)$, spectra by minimizing the penalty function (Eq. 8) in a square law sense.

$$
E_{\mathrm{I}}^{\mathrm{N}}(n)=\frac{I_{\mathrm{x}}^{\mathrm{N}, \mathrm{MC}}(n)}{\left\langle I_{x}^{N, M C}(n)\right\rangle_{n}} / \frac{I_{\mathrm{x}}^{\mathrm{N}, \mathrm{M}}(n)}{\left\langle I_{\mathrm{x}}^{\mathrm{N}, \mathrm{M}}(n)\right\rangle_{n}}-1
$$

The channels, $n$, included in the penalty function are restricted to the ones above $500 \mathrm{~nm}$, since they are not well modelled by our inverse Monte Carlo model for lower wavelengths. This can be seen in Figures 3 and 4 .

To make the estimation more sensitive to oxygen saturation, an extra term, $E_{\mathrm{s}}^{\mathrm{N}}$, is added to the penalty function. It consists of the ratio between four of the channels in the region 540-580 $\mathrm{nm}$ where the differences between oxygenated and deoxygenated blood is large, see Figure 2. The ratio, presented in Eq. 9, is designed to have minimal impact from skin model parameters not related to blood. 


$$
E_{\mathrm{S}}^{\mathrm{N}}=\frac{I_{\mathrm{X}}^{\mathrm{N}}(557)+I_{\mathrm{X}}^{\mathrm{N}}(570)}{I_{\mathrm{X}}^{\mathrm{N}}(544)+I_{\mathrm{X}}^{\mathrm{N}}(580)}
$$

This gives us a new penalty function described in Eq. 10. The extra term is multiplied by ten to give enhanced impact on the calculated error.

$$
E^{\mathrm{N}}=\left[\begin{array}{c}
E_{\mathrm{I}}^{\mathrm{N}}(n) \\
10 \cdot E_{\mathrm{S}}^{\mathrm{N}}
\end{array}\right]
$$

The algorithm then finds the best fit in a least-square sense as in Eq. 11.

$$
\min \left\|E^{\mathrm{N}}\right\|_{2}^{2}
$$
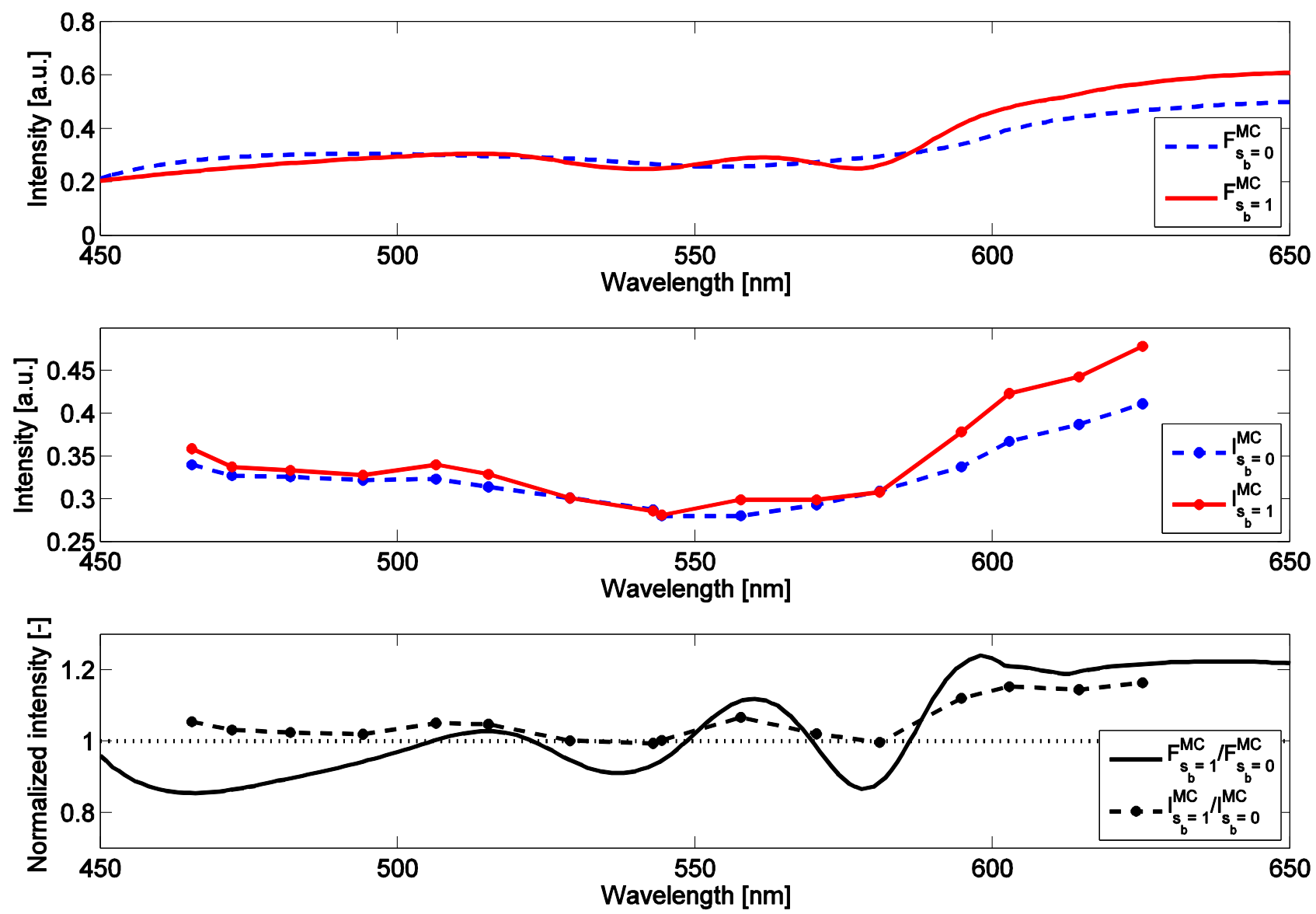

Figure 2. Upper panel: Monte Carlo modelled spectra of desaturated blood (blue dashed line) and fully saturated blood (red solid line). Other parameters are set to their mid-range values (see Table 1). Mid-panel: Monte Carlo modelled spectra of desaturated blood (blue dashed line) and fully saturated blood (red solid line) compensated for sensitivity of each of the 16 channels (marked with dots) of the xiSpec detector. Other parameters are set to their mid-range values. Lower panel: The ratio of the two respective spectra in the upper (black solid line) and middle (black dashed line) panels. 


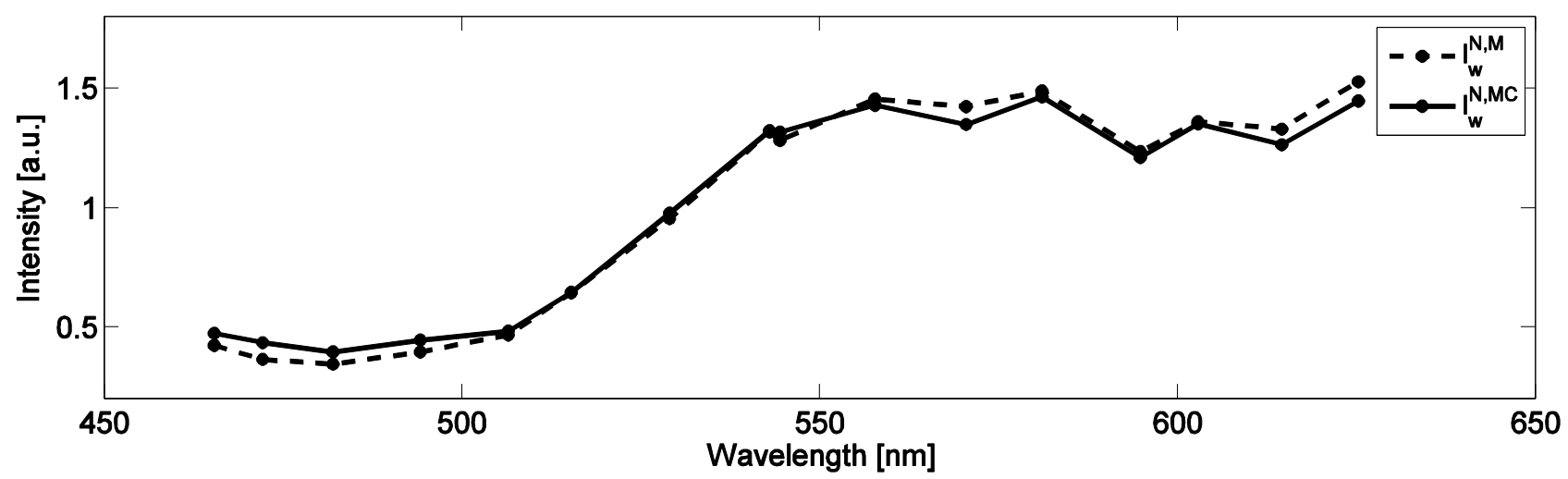

Figure 3. Measured (black dashed line) vs modelled (black solid line) white spectrum for the 16 channels (marked by dots) of the xiSpec detector.
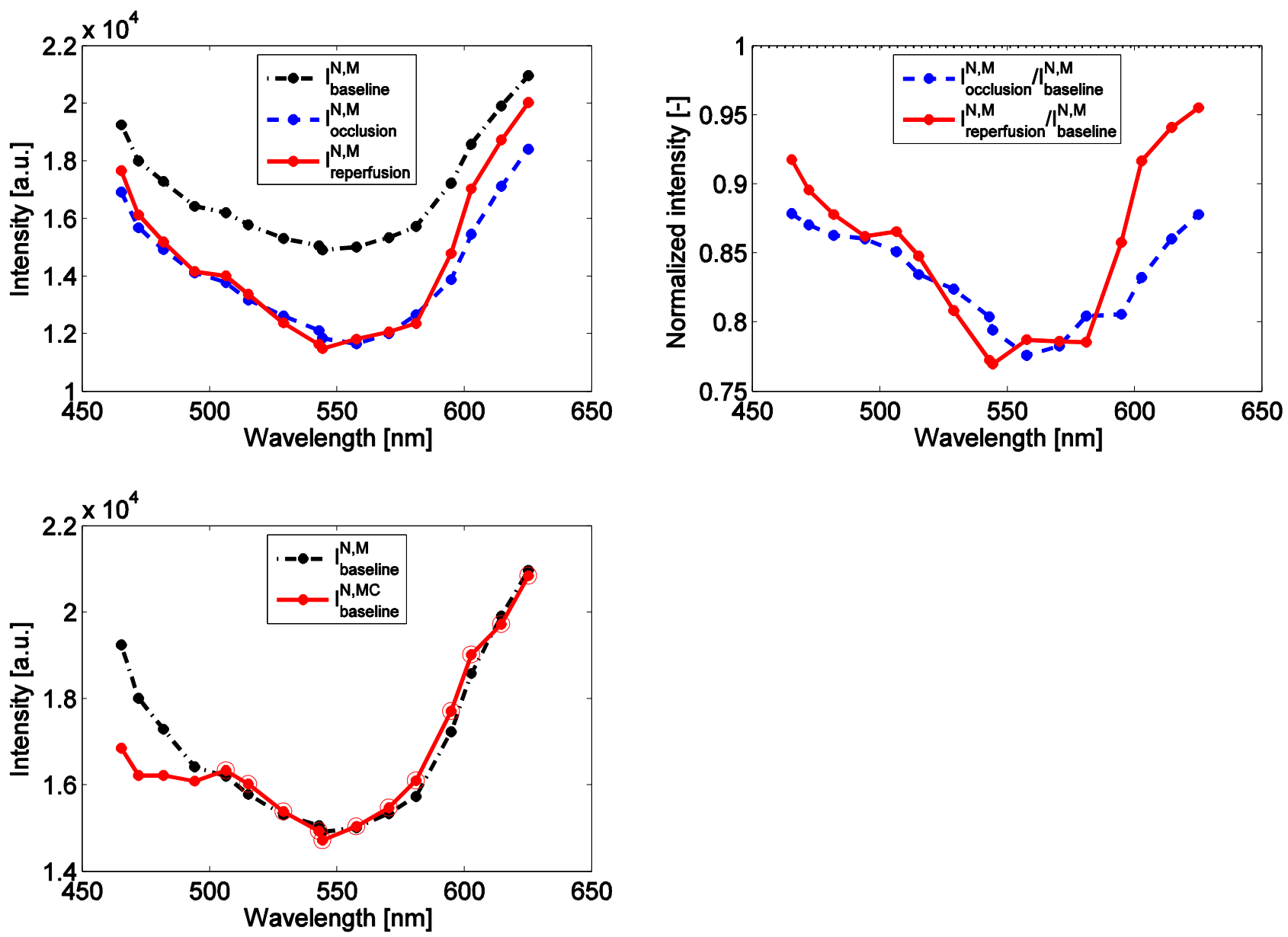

Figure 4. Upper left panel: Acquired data for the 16 channels (marked with dots) at baseline ( $\mathrm{t}=1.0$ minutes, black dash-dotted line), at the end of arterial occlusion $(t=9.5$ minutes, blue dashed line $)$ and after release of the cuff $(t=10.2$ minutes, red solid line). Upper right panel: Comparison to baseline (ratio) of data acquired after 4.5 minutes of occlusion ( $\mathrm{t}=9.5$ minutes, blue dashed line) and after release $(\mathrm{t}=10.2$ minutes, red solid line). Lower left panel: Comparison between measured (black dash-dotted line) and modelled (red solid line) spectrum at baseline $\left(\mathrm{t}=1.0\right.$ minutes). Red encircled dots mark channels, $n$, used for the penalty function, $E_{\mathrm{I}}^{\mathrm{N}}(n)$. 


\section{RESULTS}

To validate the spectral response of the sensor, the LED spectrum and the bio-optical skin tissue model (Eq. 5 and Eq. 6), measured and modelled white spectra are compared in Figure 3. In Figure 4, measured data at three points of time are presented; during baseline before occlusion ( $\mathrm{t}=1.0$ minutes $)$, at the end of arterial occlusion $(\mathrm{t}=9.5$ minutes $)$ and right after release of the cuff ( $t=10.2$ minutes). The measured data in baseline is also presented together with the spectrum modelled by the inverse Monte Carlo algorithm for comparison.

In Figure 5, the calculated oxygen saturation (arterial occlusion protocol) and blood volume (venous occlusion protocol) are depicted. EPOS data is presented as reference. Results from both Model A (parameters $c_{\mathrm{b}}, s_{\mathrm{b}}, c_{\mathrm{mel}}$ are set free) and B (parameters $c_{\mathrm{b}}, s_{\mathrm{b}}, c_{\mathrm{mel}}, \alpha, \beta, T_{\mathrm{epi}}$ are set free) are shown for comparison on the performance of the algorithm with three and six free parameters respectively. The oxygen saturation and blood volume are estimated based on the average intensity over a $2 \times 2 \mathrm{~cm}$ large area of interest (marked by a white square in Figure 6).

The oxygen saturation maps in Figure 6, showing the spatial oxygenation of the lower arm, are calculated for each pixel by the inverse Monte Carlo algorithm during baseline $(\mathrm{t}=1.0$ minutes $)$, arterial occlusion $(\mathrm{t}=9.5$ minutes $)$ and release $(\mathrm{t}$ $=10.2$ minutes).
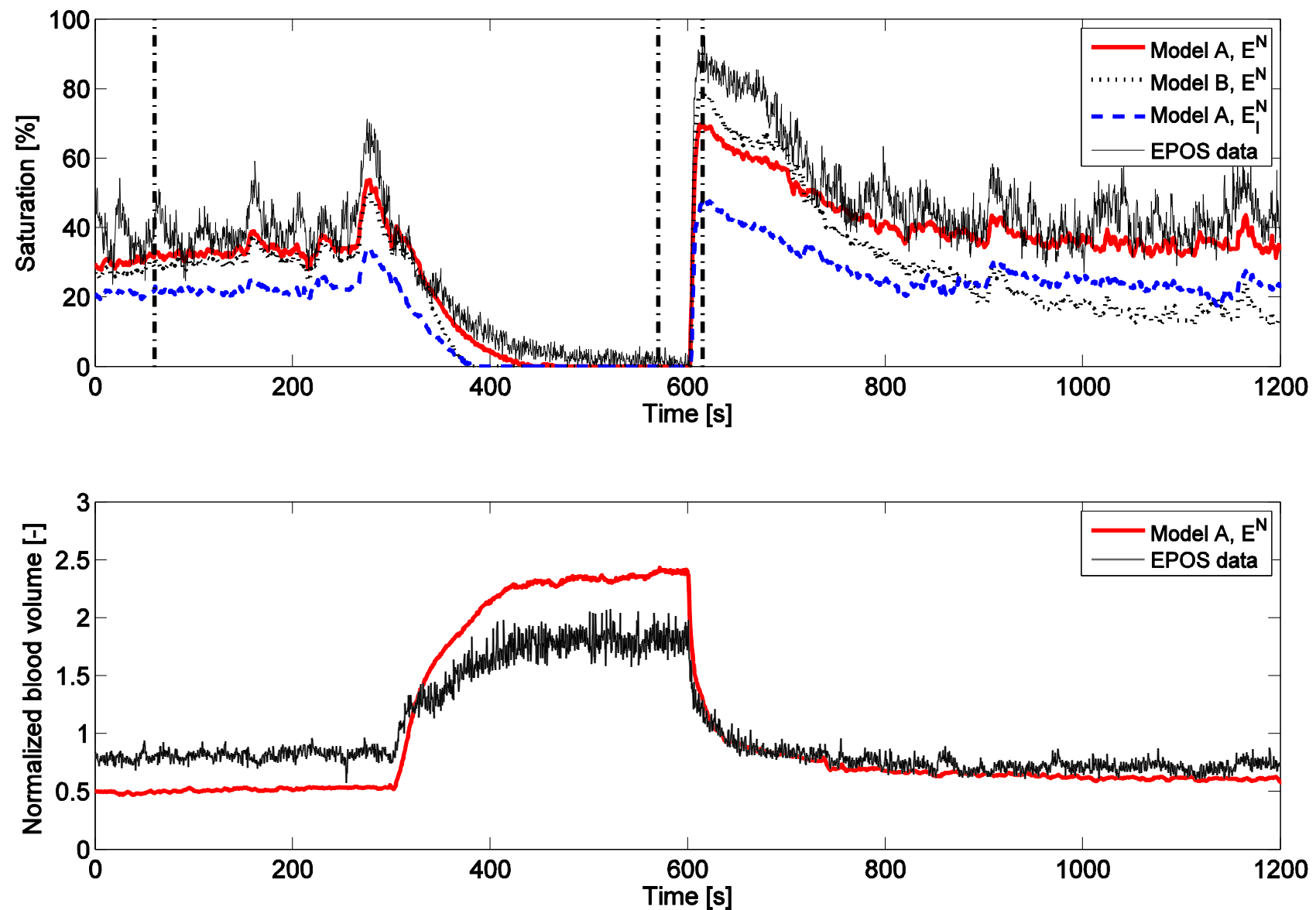

Figure 5. Estimated oxygen saturation during arterial occlusion (upper panel) and blood volume during venous occlusion (lower panel), using the inverse Monte Carlo algorithm on the average detected intensity in an area of interest (the white square in Figure 6). For arterial occlusion, the inverse Monte Carlo algorithm using Model A (three free parameters, red solid line) is compared to the result using Model $\mathrm{B}$ (letting all six parameters free, black dotted line) and to the result when not including the oxygen saturation sensitive index $E_{\mathrm{S}}^{\mathrm{N}}$ in the penalty function (blue dashed line). As reference the respective oxygen saturation and blood volume measured simultaneously with the EPOS system is presented (black solid line). Vertical dash-dotted lines show the points in time during arterial occlusion protocol, for which spatial calculations are presented in Figure 6. 

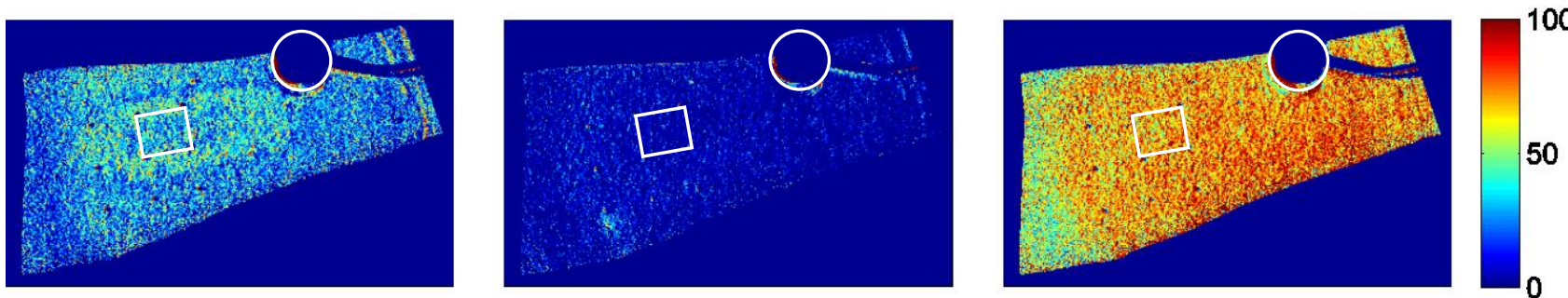

Figure 6. From left to right: Oxygen saturation maps at baseline $(t=1.0$ minutes $)$, at the end of arterial occlusion $(t=9.5$ minutes $)$ and after release of the cuff $(\mathrm{t}=10.2$ minutes) calculated by the inverse Monte Carlo algorithm. A white square borders the area of interest for the temporal measurements (Figure 5) and the position of the EPOS system probe is marked by a white circle.

\section{DISCUSSION}

We have used a snapshot imager, the xiSpec camera, having 16 wavelength-specific Fabry-Perot filters overlaid on a custom CMOS-chip, and revealed possibilities to explore oxygenation and blood volume temporally and spatially, with prospects to monitor physiological changes.

The white spectrum measured with the xiSpec camera, $I_{\mathrm{w}}^{\mathrm{N}, \mathrm{M}}(n)$, compared to the Monte Carlo modelled white spectrum, $I_{\mathrm{w}}^{\mathrm{N}, \mathrm{MC}}(n)$, showed high conformity in the range 500 to $630 \mathrm{~nm}$, although the channels specific spectral response is overlapping and sometimes show multimodal distributions (see Figures 3 and 1 respectively). This conformity strongly suggests that the proposed way of modelling the camera response and the LED set-up can be used in an inverse Monte Carlo algorithm. To further improve the similarity between measured and modelled spectra, all spectra are white normalized before being compared. Applying the inverse Monte Carlo algorithm to measured and white normalized spectra also display that the method is capable of accurately model spectra from real skin tissue.

Differences appearing could originate from the detector sensitivity given by the manufacturer. Changing the numerical aperture of the lens mounted on the xiSpec camera slightly changes the spectral response of the channels, which most likely will affect the precision of determining the blood oxygenation. The detector sensitivity should optimally be measured for the specific lens-sensor set-up used. The impact of the light source is accepted to have less influence since the LED emission spectrum display only small variations with no characteristic spectral features for wavelengths longer than 500 $\mathrm{nm}$.

Spectral mismatch can also be attributed to the bio-optical model. All measurements include a polarising filter on the xiSpec camera that has not been implemented in the Monte Carlo modelling. This should not affect the white reference measurement but might impact skin tissue measurements. The light transport model, used for Monte Carlo simulations, is further limited as it includes only a finite number of parameters and is not a full representation of the complex reality. Still, our results show that a good spectral fit can be attained using only three free parameters.

Our set-up detects a decrease in intensity, which is related to the characteristic blood absorption spectra, when comparing baseline to occluded and re-perfused tissue in Figure 4. This is likely due to a re-distribution of blood, from deeper, larger arterioles to more superficial capillaries and venules, during the occlusion phase. During the re-perfusion phase, the decreased detected intensity is associated with large inflow of oxygenated blood from the post-occlusive hyperaemic reaction. The measured intensity changes are rather small as blood oxygenation goes from a low to a high value (from occluded to re-perfused tissue) reflected in a transition from deoxyhaemoglobin to oxyhaemoglobin. Despite this, the inverse Monte Carlo algorithm is sensitive enough to model the measured spectrum, enabling small changes in tissue physiology to be monitored and displayed.

For evaluation of the inverse Monte Carlo algorithm, the two models presented in Table 1 are tested. Figure 5 show that the oxygen saturation sensitive penalty function, including $E_{\mathrm{S}}^{\mathrm{N}}$, increases, as predicted, the accuracy of the oxygen saturation estimation in relation to the reference EPOS measurements. The added penalty term significantly reduces the computational time. The results suggest that Model A (three free parameters), compared to Model B (six free parameters), 
performs equally well, or even better, and also requires significantly less computational time. Therefore, we use model A for further calculations. The estimated oxygen saturation and blood volume values reflect the expected trends following both arterial and venous occlusion ${ }^{12}$.

The EPOS system measures pointwise DRS adjacent to the area evaluated by our inverse Monte Carlo algorithm, but for practical reasons it is not possible to evaluate the same area for both methods at the same time. Still, the oxygen saturation estimations of both modalities display great similarities, even though different areas are examined. This indicates that the spatial differences in the true oxygen saturation is relatively small, which is also supported by the low spatial oxygen variability depicted in Figure 6, at least for the forearm. To further evaluate the consistency of these results, an extended number of test subjects and measurements needs to be analysed.

The penetration depth of the detected photons may also vary between the two modalities, which likely affects the result. This difference could explain the relatively large deviation in estimated blood volume during venous occlusion (Figure 5, lower panel). Therefore, to properly evaluate the accuracy of our method, phantoms with known blood amount and oxygenation levels are needed, as there exist no gold-standard method for skin measurements at an identical sampling volume.

When calculating the oxygenation pixel-wise and spatially for the area of the forearm, the change in oxygen saturation between baseline, occlusion and re-perfusion is clearly visible. The spatial variability, similar to image shot noise, could either originate from true variations in the microcirculation or, as indicated by the "hot" pixels in the occlusion image, from system noise, calibration errors or calculation errors. To cancel out non-physiological noise, we use the average value over an area of interest for the temporal calculations in Figure 5.

Inverse Monte Carlo modelling is computationally demanding and limit the prospect of fast on-line analysis of snapshot image data with high resolution. The processing time for a single blood oxygenation image using our algorithm was about 80 minutes. To substantially improve this, other non-iterative algorithms, tailored for a high sensitivity to blood oxygenation and blood volume changes, needs to be explored further. This could open for the possibility to accurately image physiological changes related to microcirculation in real-time.

\section{CONCLUSIONS}

We have shown that a multispectral snapshot imager with 16 strongly overlapping wavelength bands can capture the subtle spectral changes associated with the haemodynamic variations occurring during an occlusion provocation. By using an inverse Monte Carlo algorithm, taking into account the full spectral response of each band, the MSI data can be analysed to reveal changes in both blood volume and blood oxygenation, spatially and temporally, on human skin.

\section{ACKNOWLEDGEMENTS}

The authors wants to thank Tomas Strömberg (Department of Biomedical Engineering, Linköping University) for help with EPOS data extraction. This project is supported by funding from the Centre for Industrial Information Technology (CENIIT) at Linköping University in collaboration with Perimed AB, Järfälla, and by the EU FP7 project SEMEOTICONS (SEMEiotic Oriented Technology for Individual's CardiOmetabolic risk self-assessmeNt and Self-monitoring), 611516. 


\section{REFERENCES}

[1] Ellis, C. G., Jagger, J., and Sharpe, M., "The microcirculation as a functional system," Critical Care, 9, S3-S8 (2005).

[2] Intaglietta, M., Johnson, P. C., and Winslow, R. M., "Microvascular and tissue oxygen distribution," Cardiovascular Research, 32(4), 632-643 (1996).

[3] Pittman, R. N., "Oxygen transport and exchange in the microcirculation," Microcirculation, 12(1), 59-70 (2005).

[4] Alkawaz, M. H., Mohamad, D., Saba, T., Basori, A. H., and Rehman, A., "The Correlation Between Blood Oxygenation Effects and Human Emotion Towards Facial Skin Colour of Virtual Human," 3d Research, 6(2), (2015).

[5] Stephen, I. D., Coetzee, V., Smith, M. L., and Perrett, D. I., "Skin Blood Perfusion and Oxygenation Colour Affect Perceived Human Health,” Plos One, 4(4), (2009).

[6] Zijlstra, W. G., Buursma, A., and Meeuwsen-van der Roest, W. P., "Absorption spectra of human fetal and adult oxyhemoglobin, de-oxyhemoglobin, carboxyhemoglobin, and methemoglobin," Clin Chem, 37(9), 1633-8 (1991).

[7] Hernandez, S. E., Rodriguez, V. D., Perez, J., Martin, F. A., Castellano, M. A., and Gonzalez-Mora, J. L., "Diffuse reflectance spectroscopy characterization of hemoglobin and intralipid solutions: in vitro measurements with continuous variation of absorption and scattering," Journal of Biomedical Optics, 14(3), (2009).

[8] Koz'ma, S. Y., Aleksandrov, M. T., and Lebedev, A. V., "Algorithms for estimating oxygen saturation in biological tissues," Journal of Communications Technology and Electronics, 51(12), 1413-1417 (2006).

[9] Miclos, S., Parasca, S. V., Calin, M. A., Savastru, D., and Manea, D., "Algorithm for mapping cutaneous tissue oxygen concentration using hyperspectral imaging," Biomedical Optics Express, 6(9), 3420-3430 (2015).

[10] Hennessy, R., Goth, W., Sharma, M., Markey, M. K., and Tunnell, J. W., "Effect of probe geometry and optical properties on the sampling depth for diffuse reflectance spectroscopy," J Biomed Opt, 19(10), 107002 (2014).

[11] Durduran, T., Choe, R., Baker, W. B., and Yodh, A. G., "Diffuse optics for tissue monitoring and tomography," Reports on Progress in Physics, 73(7), (2010).

[12] Jonasson, H., Fredriksson, I., Pettersson, A., Larsson, M., and Stromberg, T., "Oxygen saturation, red blood cell tissue fraction and speed resolved perfusion - A new optical method for microcirculatory assessment,"

Microvascular Research, 102, 70-77 (2015).

[13] Palmer, G. M., and Ramanujam, N., "Monte Carlo-based inverse model for calculating tissue optical properties. Part I: Theory and validation on synthetic phantoms," Appl Opt, 45(5), 1062-71 (2006).

[14] Palmer, G. M., Zhu, C., Breslin, T. M., Xu, F., Gilchrist, K. W., and Ramanujam, N., "Monte Carlo-based inverse model for calculating tissue optical properties. Part II: Application to breast cancer diagnosis," Appl Opt, 45(5), 1072-8 (2006).

[15] Zhu, C. G., and Liu, Q., "Hybrid method for fast Monte Carlo simulation of diffuse reflectance from a multilayered tissue model with tumor-like heterogeneities," Journal of Biomedical Optics, 17(1), (2012).

[16] Holmer, A., Tetschke, F., Marotz, J., Malberg, H., Markgraf, W., Thiele, C., and Kulcke, A., "Oxygenation and perfusion monitoring with a hyperspectral camera system for chemical based tissue analysis of skin and organs," Physiological Measurement, 37(11), (2016).

[17] Jolivot, R., Benezeth, Y., and Marzani, F., "Skin parameter map retrieval from a dedicated multispectral imaging system applied to dermatology/cosmetology,” Int J Biomed Imaging, 2013, 978289 (2013).

[18] Nishidate, I., Wiswadarma, A., Hase, Y., Tanaka, N., Maeda, T., Niizeki, K., and Aizu, Y., "Noninvasive spectral imaging of skin chromophores based on multiple regression analysis aided by Monte Carlo simulation," Optics Letters, 36(16), 3239-3241 (2011).

[19] Nishidate, I., Tanaka, N., Kawase, T., Maeda, T., Yuasa, T., Aizu, Y., Yuasa, T., and Niizeki, K., "Noninvasive imaging of human skin hemodynamics using a digital red-green-blue camera," Journal of Biomedical Optics, 16(8), (2011).

[20] Lu, G., and Fei, B., "Medical hyperspectral imaging: a review," J Biomed Opt, 19(1), 10901 (2014).

[21] Cuccia, D. J., Bevilacqua, F., Durkin, A. J., Ayers, F. R., and Tromberg, B. J., "Quantitation and mapping of tissue optical properties using modulated imaging," Journal of Biomedical Optics, 14(2), (2009). 
[22] Huynh, C. P., and Robles-Kelly, A., "A Comparative Evaluation of Spectral Reflectance Representations for Spectrum Reconstruction, Interpolation and Classification," 2013 Ieee Conference on Computer Vision and Pattern Recognition Workshops (Cvprw), 328-335 (2013).

[23] Kainerstorfer, J. M., Ehler, M., Amyot, F., Hassan, M., Demos, S. G., Chernomordik, V., Hitzenberger, C. K., Gandjbakhche, A. H., and Riley, J. D., "Principal component model of multispectral data for near real-time skin chromophore mapping," Journal of Biomedical Optics, 15(4), (2010).

[24] Kainerstorfer, J. M., Riley, J. D., Ehler, M., Najafizadeh, L., Amyot, F., Hassan, M., Pursley, R., Demos, S. G., Chernomordik, V., Pircher, M., Smith, P. D., Hitzenberger, C. K., and Gandjbakhche, A. H., "Quantitative principal component model for skin chromophore mapping using multi-spectral images and spatial priors," Biomed Opt Express, 2(5), 1040-58 (2011).

[25] Nishidate, I., Maeda, T., Niizeki, K., and Aizu, Y., "Estimation of melanin and hemoglobin using spectral reflectance images reconstructed from a digital RGB image by the Wiener estimation method," Sensors (Basel), 13(6), 7902-15 (2013).

[26] Ewerlof, M., Salerud, E. G., Stromberg, T., and Larsson, M., "Estimating skin blood saturation by selecting a subset of hyperspectral imaging data," Imaging, Manipulation, and Analysis of Biomolecules, Cells, and Tissues Xiii, 9328, (2015).

[27] Klaessens, J. H. G. M., Noordmans, H. J., de Roode, R., and Verdaasdonk, R. M., "Non-invasive skin oxygenation imaging using a multi-spectral imaging system, Effectiveness of different concentration algorithms applied on human skin," World Congress on Medical Physics and Biomedical Engineering, Vol 25, Pt 2 Diagnostic Imaging, 25, 725-728 (2009).

[28] Rodriguez-Diaz, E., Velez-Reyes, M., Chin, R. K., and DiMarzio, C. A., "Wavelength selection for imaging hemoglobin in skin," Subsurface Sensing Technologies and Applications Ii, 4129, 243-248 (2000).

[29] Hagen, N., and Kudenov, M. W., "Review of snapshot spectral imaging technologies," Optical Engineering, 52(9), (2013).

[30] Fredriksson, I., Burdakov, O., Larsson, M., and Stromberg, T., "Inverse Monte Carlo in a multilayered tissue model: merging diffuse reflectance spectroscopy and laser Doppler flowmetry," Journal of Biomedical Optics, 18(12), (2013).

[31] Jacques, S. L., "Optical properties of biological tissues: a review,” Phys Med Biol, 58(11), R37-61 (2013). 